\title{
Racial and Ethnic Disparities in Hospital Admissions from COVID-19: Determining the Impact of Neighborhood Deprivation and Primary Language
}

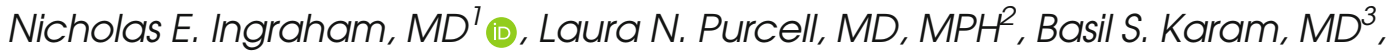 \\ R. Adams Dudley, MD, MBA' , Michael G. Usher, MD, PhD4, \\ Christopher A. Warlick, MD, PhD ${ }^{5}$, Michele L. Allen, MD, $M S^{6}$, \\ Genevieve B. Melton, MD, PhD 7,8 , Anthony Charles, MD, FACS, MPH ${ }^{2,9}$, and \\ Christopher J. Tignanelli, $M D^{7,8,10}$
}

\begin{abstract}
'Department of Medicine, Division of Pulmonary and Critical Care, University of Minnesota, Minneapolis, MN, USA; ${ }^{2}$ Department of Surgery, University of North Carolina, Chapel Hill, NC, USA; ${ }^{3}$ Department of Surgery, Medical College of Wisconsin, Milwaukee, WI, USA; ${ }^{4}$ Department of Medicine, Division of General Internal Medicine, University of Minnesota, Minneapolis, MN, USA; ${ }^{5}$ Department of Urology, University of Minnesota, Minneapolis, MN, USA; 'Department of Family Medicine and Community Health, University of Minnesota, Minneapolis, MN, USA; ${ }^{7}$ Institute for Health Informatics, University of Minnesota, Minneapolis, MN, USA; ${ }^{8}$ Department of Surgery, University of Minnesota, Minneapolis, MN, USA; ${ }^{5}$ School of Public Health, University of North Carolina, Chapel Hill, NC, USA; ${ }^{10}$ Department of Surgery, North Memorial Health Hospital, Robbinsdale, MN, USA.
\end{abstract}

BACKGROUND: Despite past and ongoing efforts to achieve health equity in the USA, racial and ethnic disparities persist and appear to be exacerbated by COVID-19.

OBJECTIVE: Evaluate neighborhood-level deprivation and English language proficiency effect on disproportionate outcomes seen in racial and ethnic minorities diagnosed with COVID-19.

DESIGN: Retrospective cohort study

SETTING: Health records of 12 Midwest hospitals and 60 clinics in Minnesota between March 4, 2020, and August 19, 2020

PATIENTS: Polymerase chain reaction-positive COVID19 patients

EXPOSURES: Area Deprivation Index (ADI) and primary language

MAIN MEASURES: The primary outcome was COVID-19 severity, using hospitalization within 45 days of diagnosis as a marker of severity. Logistic and competing-risk regression models assessed the effects of neighborhoodlevel deprivation (using the $\mathrm{ADI}$ ) and primary language. Within race, effects of ADI and primary language were measured using logistic regression.

Nicholas E. Ingraham, Laura N. Purcell, Anthony Charles and Christopher J. Tignanelli contributed equally to this work.

Key Points

- Guestion: Do socioeconomic factors or primary language account for racial disparities in COVID-19 disease severity?

- Findings: In this observational study of 5577 adults, race, ethnicity, and non-English-speaking as a primary language are associated with increased risk of severe COVID-19 disease, independent of neighborhood-level deprivation.

- Meaning: Racial and ethnic disparities related to COVID-19 severity are not explained by socioeconomic factors, which supports further investigation into other confounders (e.g., testing, community outreach, racism) and highlights the need to focus on our non-English-speaking populations.

Received September 28, 2020

Accepted April 1, 2021

Published online May 18, 2021
RESULTS: A total of 5577 individuals infected with SARS-CoV-2 were included; 866 ( $n=15.5 \%$ ) were hospitalized within 45 days of diagnosis. Hospitalized patients were older (60.9 vs. 40.4 years, $p<0.001$ ) and more likely to be male ( $n=425$ [49.1\%] vs. 2049 [43.5\%], $p=0.002$ ). Of those requiring hospitalization, $43.9 \%$ ( $n=381), 19.9 \%$ $(n=172), 18.6 \%(n=161)$, and $11.8 \%(n=102)$ were White, Black, Asian, and Hispanic, respectively. Independent of ADI, minority race/ethnicity was associated with COVID-19 severity: Hispanic patients (OR 3.8, 95\% CI 2.72-5.30), Asians (OR 2.39, 95\% CI 1.74-3.29), and Blacks (OR 1.50, 95\% CI 1.15-1.94). ADI was not associated with hospitalization. Non-English-speaking (OR $1.91,95 \%$ CI 1.51-2.43) significantly increased odds of hospital admission across and within minority groups. CONCLUSIONS: Minority populations have increased odds of severe COVID-19 independent of neighborhood deprivation, a commonly suspected driver of disparate outcomes. Non-English-speaking accounts for differences across and within minority populations. These results support the ongoing need to determine the mechanisms that contribute to disparities during COVID-19 while also highlighting the underappreciated role primary language plays in COVID-19 severity among minority groups.

J Gen Intern Med 36(11):3462-70 DOI: $10.1007 /$ s11606-021-06790-w

(C) Society of General Internal Medicine 2021

\section{INTRODUCTION}

Health disparities linger in the USA, and their elimination remains an essential health and societal imperative. ${ }^{1,2}$ Concerns of racial health inequity worsening during COVID-19 were raised immediately, ${ }^{3-5}$ and first identified in a landmark study of New York City's five boroughs. ${ }^{6}$ Additionally, community-level studies demonstrated lower socioeconomic 
status (SES) was associated with the risk of SARS-CoV-2 infection. $^{7}$

The etiology of health disparities is multifactorial and includes differences in sociocultural environment, behavior, biological profile, healthcare system, and clinical events. ${ }^{8-18}$ Specific to COVID-19, multiple explanations driving health disparities have been proposed, for example, higher rates of "essential workers," multigenerational housing, increased rates of poverty, comorbidities, and uninsured status in minority populations. ${ }^{19}$ Early data regarding COVID-19's impact on health disparities are emerging, ${ }^{20-29}$ studies rarely account for multiple socioeconomic variables and granular patient-level characteristics simultaneously. Previous studies used individual-level poverty (uninsured or Medicaid enrollment or homelessness) or neighborhood-level poverty (percent of low-income residents or median neighborhood income) with variable definitions. ${ }^{29-32}$ These poverty measures may inadequately evaluate disparate outcomes. Furthermore, another evolving confounder is English language proficiency (ELP). At the county level, ELP was one of the strongest predictors of poor outcomes in COVID-19. ${ }^{33}$ Despite this association, language proficiency at the individual-level analysis remains unknown.

We hypothesized that neighborhood-level deprivation and non-English as a primary language (at the individual level) might account for the disproportionate outcomes seen in racial and ethnic minorities. Understanding these complex relationships is critical to guiding the ongoing prevention, policy, and intervention efforts to fight this global pandemic equitably. ${ }^{34}$ The purpose of this study was to evaluate the impact of neighborhood-level deprivation and primary language on disproportionate outcomes seen in racial and ethnic minorities diagnosed with COVID-19.

\section{METHODS}

\section{Design and Data}

A retrospective analysis of 12 Minnesotan hospitals and 60 primary care clinics between March 4 and August 19, 2020, was performed. We pooled data across different electronic health records (EHRs) utilizing a unique patient identifier to account for healthcare encounters across systems. The University of Minnesota institutional review board (STUDY00001489) approved and provided a waiver of consent for this study.

\section{POPULATION}

Individuals with polymerase chain reaction (PCR)-confirmed COVID-19 that did not opt out of research were included in the study. Less than $2.5 \%$ of patients opt out of research, a decision made when they establish care. Patients with missing race or ethnicity data were excluded (1225 patients, $17.9 \%$ ) or those developing new-onset COVID-19 following hospital admission (13 patients).

\section{Outcomes}

Our primary outcome was the need for hospitalization within 45 days of PCR-confirmed COVID-19 disease. By August 24, 2020 , individuals not hospitalized were censored in the competing-risk regression model and treated as not requiring hospitalization in the logistic regression model.

\section{Independent Variables/Covariates}

The independent variables for this study included race (White/ Black/Asian/Other) or ethnicity (Hispanic/non-Hispanic), English versus non-English-speaking, and the national Area Deprivation Index (ADI) categorized into quintiles (first quintile: lowest neighborhood-level deprivation, fifth quintile: highest neighborhood-level deprivation). Race and ethnicity were self-reported and collected for administrative purposes, and quality was not verified. To perform the analysis, we made a categorical variable including both race and ethnicity. We categorized patients identifying as non-Hispanic into White, Black, Asian, or Other with the understanding that race is a social construct and distinctly different from ethnicity; however, when assessing minority populations, it is prudent to include all communities that may be affected.

The ADI's development has been described previously. ${ }^{35}$ The ADI is a measure of the socioeconomic disadvantage of a neighborhood (United States Census Block Groups) calculated from 17 different indicators that include education, housing (e.g., occupancy rates, household value), poverty (e.g., median income, number of dependents, \% with complete plumbing), and employment status. $\mathrm{ADI}^{36}$ and other indices ${ }^{37-39}$ are utilized to estimate these socioeconomic variables' effects on aggregate outcomes, including in COVID-19.

All models were adjusted for age, gender, Elixhauser Comorbidity Index, ${ }^{40}$ relationship status, and rurality/urbanity. Urban was defined as living in a zip code with a population density $>200$ people per square mile. ${ }^{41}$ Marital status was characterized as single, married (domestic partner, life partner), divorced, or widowed.

\section{Statistical Analysis}

Median and interquartile range (IQR) expressed the central tendency for continuous variables with a skewed distribution. Categorical variables' frequencies were described as percentages. The bivariate analysis compared those requiring and not requiring hospital admission. The association of race and ethnicity on mortality, hospital admission severity (laboratory values/vitals), in-hospital complications, intensive care unit (ICU) and mechanical ventilation utilization, and in-hospital mortality was evaluated for patients requiring hospital admission. Overall missingness was low (0$2.06 \%$ ) and imputation was deemed unnecessary. ${ }^{42}$ 
Logistic and competing-risk regression models (censored at 45 days while accounting for the competing risk of death before hospitalization) were used to assess the independent association of race/ethnicity, ADI, and primary language (English vs. other) on the primary outcome. Comorbidities were determined using a random forest variable importance analysis. Models were compared using comorbidities selected by variable importance analysis (Appendix Table 1) to aggregate indices of comorbidities commonly used to adjust for baseline conditions (Elixhauser Comorbidity Index) given the limited experience of adjusting for comorbid conditions in COVID-19. We used the best Bayesian Information Criterion (BIC) to determine the final model.

We investigated ADI and primary language effects, across and between race/ethnic groups, on the risk for admission. By plotting the cumulative incidence of hospital admission, it was clear there were consistent and sequential increases across and within groups in relation to both ADI and primary language. To determine significance within racial and ethnic groups, logistic regression analyses (using the same covariates as the primary model) were performed by stratifying either ADI or primary language by race and ethnicity. Each model used the race and ethnicity of interest as the base to determine if ADI or language differed. For ADI models, we report the comparison between the fifth to the first ADI quintile.

Statistical analyses were performed using Stata MP, version 16 (StataCorp, College Station, TX). Statistical significance was defined as a two-tailed $p$ value $<0.05$.

\section{RESULTS}

Of the 5577 individuals infected with SARS-CoV-2 meeting inclusion criteria for this study, $866(n=15.5 \%)$ patients were admitted to the hospital within 45 days of COVID-19 diagnosis (Appendix Figure 1). Overall, the median age was 43.7 years (IQR: 27.4-62.3) and 44.4\% $(n=2474)$ were male. Patients were predominantly White $(n=2931,52.6 \%)$, followed by Black $(n=$ $1225,22.0 \%)$, Asian $(n=677,12.2 \%)$, and Hispanic $(n=416$, $7.5 \%$ ). The median national ADI was $37 \%$ (IQR: 22-48\%).

Patients who required hospitalization were older $(60.9$ years [IQR: 45.7-75.9] vs. 40.4 year [IQR: 25.6-58.3], $p<0.001)$ and a greater proportion were male $(n=425,49.1 \%$ vs. $n=$ $2049,43.5 \%, p=0.002$ ) (Table 1 ). A higher proportion of non-English-speaking patients required admission $(n=301$, $34.8 \%$ vs. $n=785,16.7 \%, p<0.001$ ). Admitted patients had higher median Elixhauser Comorbidity Index (5.0, IQR: 3.0 9.0 vs. 1.0 , IQR: $0.0-2.0, p<0.001)$ and mortality $(n=108$, $12.5 \%$ vs. $n=68,1.4 \%, p<0.001)$ than those not requiring admission.

Of those requiring hospitalization, $43.9 \%(n=381), 19.9 \%(n$ $=172), 18.6 \%(n=161)$, and $11.8 \%(n=102)$ were White, Black, Asian, and Hispanic, respectively (Appendix Table 2). White patients (69.6 years) were significantly older than their Black (55.4 years), Asian (58.9 years), and Hispanic (48.5 years) counterparts $(p<0.001)$. Approximately half of White and
Hispanic patients lived in the first and second quintile ADI neighborhoods. In contrast, nearly the same proportion of Asian patients lived in the fourth quintile, and Black patients were evenly distributed over all ADI quintiles. Admission labs, including those associated with mortality, were not significantly different between racial/ethnic groups (Appendix Table 2). ${ }^{43,44}$

Logistic regression models with Elixhauser Comorbidity Index performed better (BIC 3686) compared to using selected comorbidities (BIC 4008) as described above. Thus, the final model included Elixhauser Comorbidity Index in attempts to adjust for comorbidities.

Table 1 Univariate Analysis of Individuals Infected with SARSCoV-2 Not Admitted Compared to Those Admitted to the Hospital

\begin{tabular}{|c|c|c|c|}
\hline & $\begin{array}{l}\text { Not admitted } \\
(n=4711, \\
84.5 \%)\end{array}$ & $\begin{array}{l}\text { Admitted }(n= \\
866,15.5 \%)\end{array}$ & $\begin{array}{l}p \\
\text { value }\end{array}$ \\
\hline $\begin{array}{l}\text { Age (years): median } \\
\text { (IQR) }\end{array}$ & $\begin{array}{l}40.4(25.6- \\
58.3)\end{array}$ & $\begin{array}{l}60.9(45.7- \\
75.9)\end{array}$ & $<0.001$ \\
\hline Male sex: $n(\%)$ & 2049 (43.5) & $425(49.1)$ & 0.002 \\
\hline \multicolumn{4}{|l|}{ Race/ethnicity: $n(\%)$} \\
\hline White & $2550(54.1)$ & $381(44.0)$ & \multirow[t]{6}{*}{$<0.001$} \\
\hline Black & $1053(22.4)$ & $172(19.9)$ & \\
\hline Asian & $516(11.0)$ & $161(18.6)$ & \\
\hline Hispanic & $314(6.7)$ & $102(11.8)$ & \\
\hline Declined & 219 (4.6) & $32(3.7)$ & \\
\hline Other & $59(1.3)$ & $18(2.1)$ & \\
\hline $\begin{array}{l}\text { Non-English-speaking: } \\
n(\%)\end{array}$ & $785(16.7)$ & $301(34.8)$ & $<0.001$ \\
\hline \multicolumn{4}{|c|}{ Area deprivation quintiles: $n(\%)$} \\
\hline First: $0-20 \%$ & $1005(21.3)$ & $169(19.5)$ & \multirow[t]{5}{*}{$<0.001$} \\
\hline Second: $21-40 \%$ & $1559(33.1)$ & $237(27.4)$ & \\
\hline Third: $41-60 \%$ & $1155(24.5)$ & $220(25.4)$ & \\
\hline Fourth: $61-80 \%$ & $657(13.9)$ & $142(16.4)$ & \\
\hline Fifth: $81-100 \%$ & $335(7.1)$ & $98(11.3)$ & \\
\hline Urban: $n(\%)$ & $3416(85.8)$ & $728(93.7)$ & $<0.001$ \\
\hline \multicolumn{4}{|l|}{ Marital status: $n(\%)$} \\
\hline Single & $2428(51.5)$ & $324(37.4)$ & \multirow[t]{4}{*}{$<0.001$} \\
\hline Married & $1837(39.0)$ & $381(44.0)$ & \\
\hline Separated/divorced & $236(5.0)$ & $73(8.4)$ & \\
\hline Widowed & $210(4.5)$ & $88(10.2)$ & \\
\hline \multicolumn{4}{|l|}{ Comorbidities: $n(\%)$} \\
\hline $\begin{array}{l}\text { Hypercoagulable } \\
\text { state }\end{array}$ & $39(0.8)$ & $33(3.8)$ & $<0.001$ \\
\hline Hypocoagulable state & $211(4.5)$ & $242(27.9)$ & $<0.001$ \\
\hline $\begin{array}{l}\text { Type } 1 \text { diabetes } \\
\text { mellitus }\end{array}$ & $95(2.0)$ & $84(9.7)$ & $<0.001$ \\
\hline $\begin{array}{l}\text { Type } 2 \text { diabetes } \\
\text { mellitus }\end{array}$ & $578(12.4)$ & $338(39.0)$ & $<0.001$ \\
\hline $\begin{array}{l}\text { Atrial fibrillation or } \\
\text { atrial flutter }\end{array}$ & $220(4.7)$ & $163(18.8)$ & $<0.001$ \\
\hline Hypertension & $1,333(28.6)$ & $574(66.3)$ & $<0.001$ \\
\hline $\begin{array}{l}\text { Chronic kidney } \\
\text { disease }\end{array}$ & $334(7.2)$ & $241(27.8)$ & $<0.001$ \\
\hline $\begin{array}{l}\text { Chrease } \\
\text { Chronic obstructive } \\
\text { pulmonary disease }\end{array}$ & $169(3.6)$ & $123(14.2)$ & $<0.001$ \\
\hline $\begin{array}{l}\text { pulmonary disease } \\
\text { Liver disease }\end{array}$ & $237(5.1)$ & $131(15.1)$ & $<0.001$ \\
\hline $\begin{array}{l}\text { Cerebral vascular } \\
\text { disease }\end{array}$ & $279(6.0)$ & $151(17.4)$ & $<0.001$ \\
\hline Sleep apnea & $308(6.6)$ & $154(17.8)$ & $<0.001$ \\
\hline Congestive heart & $246(5.3)$ & $174(20.1)$ & $<0.001$ \\
\hline failure & & & \\
\hline Obesity & $849(18.2)$ & $294(33.9)$ & $<0.001$ \\
\hline Mortality: $n(\%)$ & $68(1.4)$ & $108(12.5)$ & $<0.001$ \\
\hline
\end{tabular}

Univariate analysis comparing PCR+ individuals infected with SARS$\mathrm{CoV}-2$ who were admitted within 45 days of testing vs. those without hospital admission. ADI quintiles represent lowest areas of deprivation (1st quintile) to the highest areas of deprivation (5th quintile) 


\section{Primary Analysis}

On logistic regression analysis, minority race and ethnicity were independent predictors for hospital admission (Table 2). Hispanic patients (OR 3.8, 95\% CI 2.72-5.30), Asians (OR 2.39 , 95\% CI 1.74-3.29), and Blacks (OR 1.50, 95\% CI 1.151.94) had higher odds of hospital admission within 45 days compared to White patients. ADI was not independently associated at any quintile, while being non-English-speaking (OR 1.91, 95\% CI 1.51-2.43) was independently associated with increased odds of hospitalization compared to Englishspeaking patients. These associations remained significant in the competing-risk model (Table 2).

\section{Race/Ethnicity Area Deprivation Index}

Cumulative incidence plots were generated to visualize the disparity by race and ethnicity and ADI quintile (Fig. 1a). On logistic regression, compared to White patients living in the first $\mathrm{ADI}$ quintile, there was no statistical difference in hospitalization of White patients living in the fifth ADI quintile. In contrast, there was a sequential increase in odds of needing hospitalization of first $\mathrm{ADI}$ quintile Black (OR 1.75, 95\% CI 1.05-2.93) and fifth ADI quintile Black (OR 1.90, 95\% CI 1.13-3.19), first quintile Asian (OR 2.31, 95\% CI 1.02-5.26) and fifth ADI quintile Asian (OR 4.85, 95\% CI 1.86-12.60), and first ADI quintile Hispanic (OR 3.68, 95\% CI 1.97-6.88) and fifth ADI quintile Hispanic patients (OR 10.14, 95\% CI 3.85-26.74) when compared to White patients in the first ADI quintile (Appendix Table 3). On subgroup analysis comparing the fifth vs. first quintile within each racial and ethnic group, there was no significant difference in the odds of hospitalization (Fig. 2a).

\section{Race/Ethnicity Primary Language}

Cumulative incidence plots were generated to visualize the disparity by race and ethnicity and primary language (English vs. non-English) (Fig. 1b). Similar to above, the odds of hospitalization increased across racial and ethnicity groups when stratified by primary language (English or other) and compared to English-speaking White patients. In contrast to the ADI models, on subgroup analysis, comparing those who speak English as their primary language vs. non-Englishspeaking within each minority group, there was a significant difference in the odds of hospitalization: Black (OR 1.72, 95\% CI 1.14-2.59), Asian (OR 1.61, 95\% CI 1.0-2.6), and Hispanic (3.74, 95\% CI 2.14-6.52) (Fig. 2b).

\section{DISCUSSION}

We assessed outcomes of patients diagnosed with COVID-19 in the outpatient setting. Specifically, we evaluated the association of (1) neighborhood-level deprivation (using the area deprivation index $[\mathrm{ADI}]$ ) and (2) non-English as a primary language with the need for hospital admission within 45 days (COVID-19 severity surrogate). Race and ethnicity were associated with COVID-19 severity. Furthermore, nonEnglish-speaking was associated with increased odds of admission while neighborhood-level deprivation was not. Despite our hypothesis that neighborhood deprivation and/or primary language may account for racial differences in outcomes, race and ethnicity remained statistically significant. The disparate findings by race and ethnicity in our study are not novel in isolation; however, the persistence despite controlling for proposed inequity drivers highlights a concerning and enduring healthcare crisis. Furthermore, it supports concerns $^{12,25,45}$ that other unidentified confounders (i.e., structural racism, testing, etc.) drive the association.

\section{Race/Ethnicity}

Like prior reports, we identified persisting associations between minority communities and COVID-19 outcomes despite accounting for different socioeconomic variables. $^{30,31,37,38,46}$ Hispanic patients had the highest odds of hospitalization, followed by Asian, Black, and White patients. The increased risk among Hispanic and Black patients is well documented. ${ }^{30,31}$ Similarly, in UK studies, Asian patients had higher odds of hospitalization despite controlling for deprivation areas. ${ }^{37,38}$ Our surrounding community includes large Hmong and Somali populations which provided an opportunity to evaluate multiple minority populations. While we acknowledge that administrative data with self-reported race and ethnicity data is an oversimplification of the unique populations, it highlights the concerning degree in which multiple populations under each cohort are disproportionately affected by this pandemic. The persistence of race and ethnic effects on COVID-19 outcomes contrasts disparities in other disease processes and chronic conditions. ${ }^{47-50}$ For example, racial disparities in cancer patient outcomes can be explained by treatment received and socioeconomic status. ${ }^{47-49}$ Alternatively, with COVID-19, these persisting disparities may illuminate contributing mechanisms. For example, cancer-related factors (i.e., diagnosis, treatment) occur at different points during their care. In contrast to chronic conditions or treatment plans, the pandemic provides a raw healthcare snapshot with a less confounded arena to study disparities. Furthermore, disparate outcomes appear limited to out-of-hospital care given race, ethnicity, and socioeconomic factors were not associated with in-hospital mortality in multiple studies. ${ }^{32,51}$ This suggests pre-hospitalization solutions are likely this highest yield targets to mitigate disparities.

\section{Neighborhood-Level Deprivation}

Socioeconomic status is a driver of health disparities. ${ }^{47,49,52,53}$ ADI was used, to account for socioeconomic status, because it includes key neighborhood-level characteristics, such as poverty, housing, education, and employment factors at the neighborhood level. While it is not interchangeable with individual SES, it does incorporate a portion of a patient's socioeconomic factors and may even account for racial disparities to a greater 
Table 2 Multivariable Logistic Regression and Competing-Risk Models for Hospital Admission in Individuals Infected with SARS-CoV-2

\begin{tabular}{|c|c|c|c|c|c|c|}
\hline & Odds ratio* & $95 \% \mathrm{CI}$ & $p$ value & Standardized hazard ratio & $95 \% \mathrm{CI}$ & $p$ value \\
\hline Age & 1.02 & $1.01-1.02$ & $<0.001$ & 1.01 & $1.01-1.02$ & $<0.001$ \\
\hline Male sex & 1.28 & $1.07-1.53$ & 0.006 & 1.25 & $1.08-1.46$ & $<0.001$ \\
\hline \multicolumn{7}{|l|}{ Race/ethnicity } \\
\hline White & Ref & - & - & & & \\
\hline Black & 1.50 & $1.15-1.94$ & 0.002 & 1.31 & $1.04-1.65$ & 0.02 \\
\hline Asian & 2.39 & $1.74-3.29$ & $<0.001$ & 1.78 & $1.33-2.38$ & $<0.001$ \\
\hline Hispanic & 3.80 & $2.72-5.30$ & $<0.001$ & 3.02 & $2.31-3.95$ & $<0.001$ \\
\hline Decline & 1.82 & $1.16-2.87$ & 0.009 & 1.64 & $1.11-2.44$ & 0.01 \\
\hline Other & 2.04 & $1.04-4.00$ & 0.04 & 1.89 & $1.18-3.03$ & 0.01 \\
\hline \multicolumn{7}{|l|}{ Area deprivation quintiles } \\
\hline First: $0-20 \%$ & Ref & - & - & & & \\
\hline Second: $21-40 \%$ & 0.83 & $0.65-1.07$ & 0.2 & 0.85 & $0.69-1.04$ & 0.12 \\
\hline Third: $41-60 \%$ & 0.87 & $0.67-1.13$ & 0.3 & 0.88 & $0.71-1.1$ & 0.26 \\
\hline Fourth: $61-80 \%$ & 0.88 & $0.65-1.19$ & 0.4 & 0.87 & $0.67-1.13$ & 0.29 \\
\hline Fifth: $81-100 \%$ & 1.31 & $0.93-1.85$ & 0.1 & 1.14 & $0.84-1.54$ & 0.39 \\
\hline Rural/urban & 1.34 & $0.97-1.85$ & 0.07 & 1.29 & $0.97-1.7$ & 0.08 \\
\hline \multicolumn{7}{|l|}{ Marital status } \\
\hline Single & Ref & - & - & & & \\
\hline Married & 0.92 & $0.75-1.13$ & 0.4 & 0.98 & $0.82-1.17$ & 0.82 \\
\hline Separated & 0.68 & $0.47-0.98$ & 0.04 & 0.81 & $0.59-1.11$ & 0.19 \\
\hline Widowed & 0.60 & $0.42-0.88$ & 0.008 & 0.75 & $0.56-1.02$ & 0.07 \\
\hline Non-English-speaking & 1.91 & $1.51-2.43$ & $<0.001$ & 1.52 & $1.24-1.88$ & $<0.001$ \\
\hline Elixhauser Comorbidity Index & 1.36 & $1.32-1.40$ & $<0.001$ & 1.2 & $1.18-1.22$ & $<0.001$ \\
\hline
\end{tabular}

Multivariable logistic regression (left) with odds of hospital admission in patients with PCR+ COVID-19 diagnosis within 45 days of testing. Competing-risk model (right) with standardized hazard ratio of hospital admission in patients with PCR+ COVID-19 censored at 45 days from testing while accounting for death prior the primary endpoint. ADI quintiles represent lowest areas of deprivation (lst quintile) to the highest areas of deprivation (5th quintile)

*AUROC: 0.854

degree than individual-level SES estimates. ${ }^{54}$ Surprisingly, neighborhood-level deprivation was not associated with severe COVID-19.

Prior studies found areas of deprivation were associated with higher SARS-CoV-2 infection rates, but limited data with robust SES adjustment exists for other outcomes. $15,37-39,55,56$ Price-Haywood et al. found Black patients with COVID-19 (vs. White) had higher odds of hospital admission (OR 1.96, 95\% CI 1.62-2.37) after controlling for neighborhood-level income (OR 1.22, 95\% CI 1.04-1.43). ${ }^{32}$ Similarly, other studies found non-White populations had an increased risk of poor outcomes after adjusting for insurance and poverty. ${ }^{30,31}$ In contrast to our findings and others, Ogedegbe et al. found no difference in hospitalization between Black

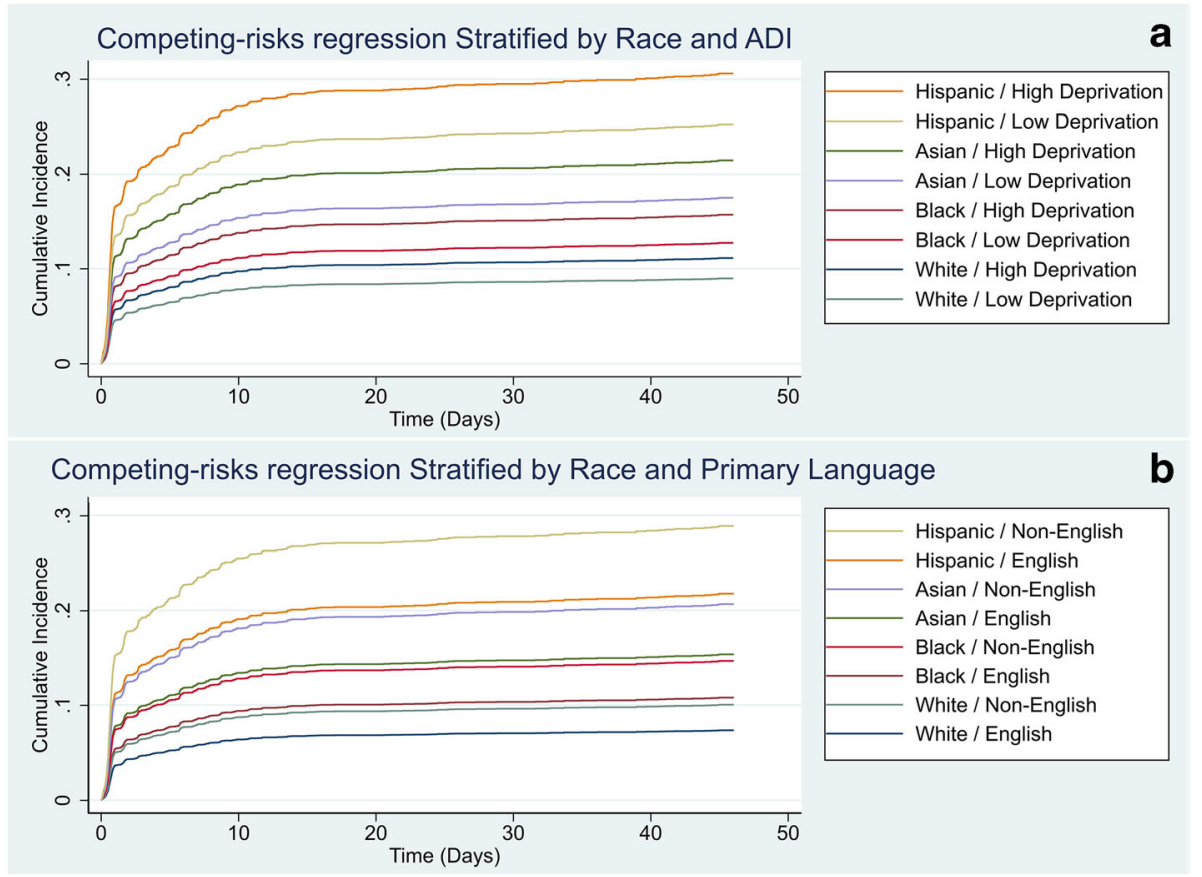

Fig. 1 Competing-risk regression cumulative incidence of hospital admission over time by ADI (a) and primary language (b) stratified by race/ethnicity. Models were censored at 45 days and accounted for death occurring prior to the primary endpoint (hospital admission). 


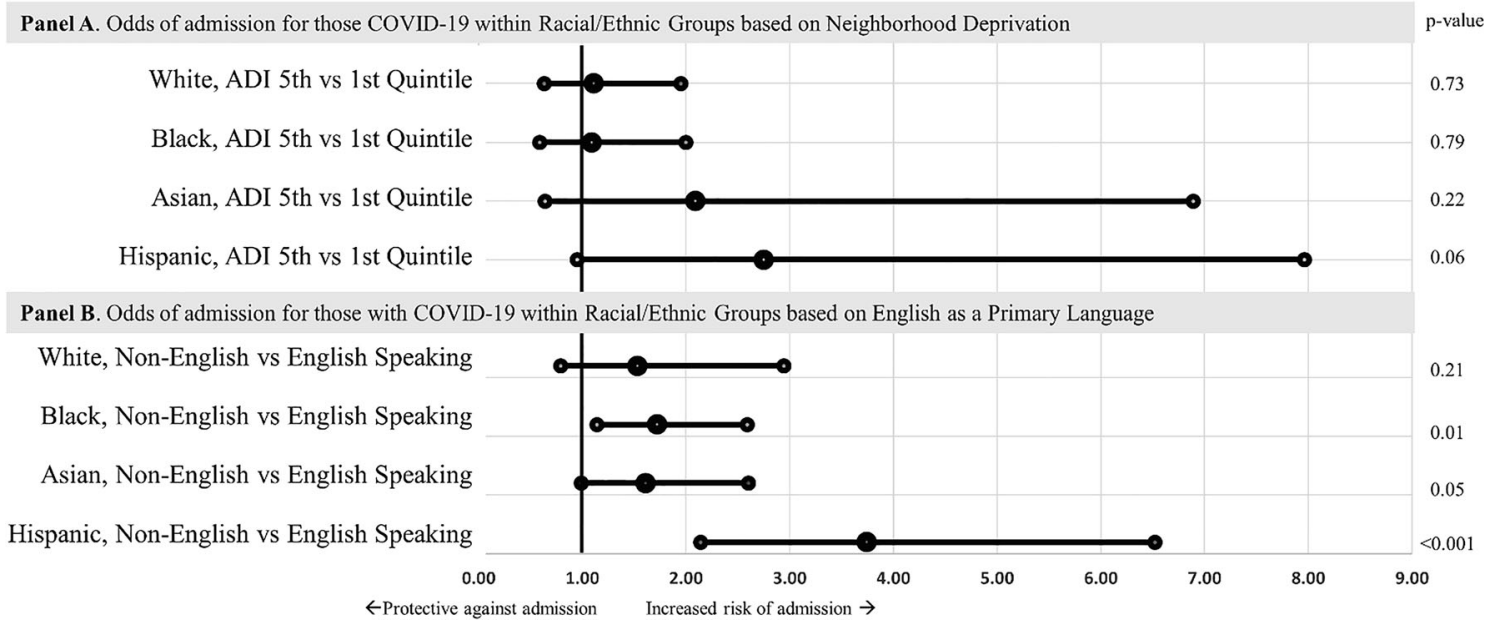

Fig. 2 Forest plots of multiple logistic regression models using each race/ethnicity as a baseline to compare ADI and primary language stratified by race/ethnicity. Multivariable logistic regression models using each race/ethnicity as a baseline within its respective model to compare within each race/ethnicity high vs. low neighborhood deprivation (top) and primary language (bottom) after adjusting for age, sex, marital status, urbanity, and comorbidities of the odds of hospital admission in patients with PCR+ COVID-19 diagnosis within 45 days of testing.

vs. White patients; however, the proportion of Black patients with insurance was significantly higher than the surrounding community rate limiting the generalizability ${ }^{56}$ Overall, these findings highlight and support the alarming fact that minorities have a higher risk of severe infection and severe disease that is not attributable solely to baseline health conditions or socioeconomic factors. Furthermore, these data suggest COVID-19 is threatening to widen the health equity gap. ${ }^{57}$

\section{Primary Language}

Unfortunately, the pandemic disproportionately affects the most vulnerable. Similar to our study, other studies found an increased risk of poor COVID-19 outcomes in non- or limitedEnglish speakers using different measures. ${ }^{20,22,33}$ Rozenfeld et al. found primary language other than English was associated with infection risk to a greater degree than race. ${ }^{22}$ Karmakar et al. recently found ELP was the most significant predictor of incidence and mortality for COVID-19 when measured at the county level. ${ }^{33}$ Strikingly, we also found non-English-speaking patients within each minority racial/ ethnic group had a higher odds of hospitalization than those who primarily spoke English. To our knowledge, this is one of the first times primary language, at the individual level within and across minority populations, was identified as a risk factor for hospitalization from COVID-19. The underappreciation of primary language as a risk factor for severe COVID-19 is likely to further these associations. English proficiency can be closely associated with socioeconomic status as it affects their daily interactions, employment opportunities, and level of discrimination. ${ }^{58}$ During the pandemic, much of the information (and misinformation) regarding public health is derived from multiple modes of media, primarily in English. ${ }^{59,60}$ While multi-lingual versions of guidelines from the CDC attempt to mitigate this shortcoming, the rapidly changing COVID-19 information makes media a more convenient avenue to consume information. Community efforts are necessary in assessing and alleviating the disparate outcomes influenced by language proficiency across and within racial and ethnic communities.

\section{Limitations}

Multiple limitations must be considered when interpreting our results. First, hospital admission criteria may vary by site. Testing deficiencies in low-risk minorities (creating the perception of higher risk) can bias the results away from the null; however, the significantly younger minority populations by $>$ 10 years (vs. White) across hospitalized patients (Appendix Table 2) suggests against the presence of this bias. Generalizability is limited beyond this population. While Minnesota has a predominantly White population, it does include the understudied Hmong and Somali populations. The analysis does not include patients who opt out of research $(<1.5 \%$ of patients) and those with missing race/ethnicity data (18\%), which may result in selection bias. Notably, these patients were missing numerous other data which limited the ability to perform multiple imputations. Insurance status (a common surrogate for SES) was not included in our analysis; however, adjustment for race, primary language, and multiple neighborhood socioeconomic attributes likely would attenuate the effects from insurance. Finally, neighborhood-level SES (ADI) and individual-level SES are sometimes discordant and attributing group-level measures to individuals may limit interpretation. ${ }^{61}$ However, in the absence of individual-level socioeconomic attributes, reliance on aggregate measures is necessary to examine these racial disparities.

\section{Next Steps}

Health equity, specifically for racial and ethnic minorities, remains an aspiration in the USA. However, failure to widely 
study $^{62,63}$ and acknowledge disparate minority outcomes ${ }^{64}$ and implement evidence-based interventions makes health equity impossible.$^{65}$ Improving access to testing is a clear first step for those communities at the highest risk. Moreover, community engagement led by trusted community representatives, from strategy to implementation, should be a universal priority. ${ }^{13}$ Minority communities, specifically non-Englishspeaking and those with lower literacy rates, are more likely to be unaware of public health recommendations and are more susceptible to misinformation, which compounds a deepseated distrust in healthcare due to historical discrimination and personal injustices. ${ }^{60,66-68}$ To combat this, we must ensure all forms of communication are available in different languages and with a robust community dissemination strategy. Urgently, we address healthcare providers' implicit bias, including trainees, to ensure healthcare provision equitably by creating a healthcare community filled with cultural humility ${ }^{69}$ Lastly, as vaccinations remain the ultimate endpoint for the pandemic, the disparate outcomes warrant our emergent attention during the vaccination prioritization process. ${ }^{65,70}$ Minority and non-English-speaking populations are clearly at high risk from multiple aspects of this health crisis, and early data already shows the maldistribution of the COVID-19 vaccine. $^{71,72}$

\section{CONCLUSION}

Our findings highlight areas of neighborhood-level deprivation may contribute to racial disparities but to a lesser degree when controlling individual-level factors. Strikingly, racial and ethnic disparities in COVID-19 illness severity exist, independent of socioeconomic characteristics, which supports the need to investigate the different levels of racism contributing to health inequity. ${ }^{73}$ Furthermore, non-ELP is associated with COVID-19 severity across and within racial and ethnic groups. We must address underlying causes of social inequalities and incorporate such actions into the national COVID-19 response in the presence of the global attention, resources, and focus in hopes of ending this pandemic closer to public health equity. ${ }^{4}$

Supplementary Information The online version contains supplementary material available at https://doi.org/10.1007/s11606-02106790-w.

Acknowledgements: The authors thank Eric Murray and the rest of the MHealth Fairview Information Technology for teaching of data management support.

Corresponding Author: Nicholas E. Ingraham, MD; Department of Medicine, Division of Pulmonary and Critical Care, University of Minnesota, Minneapolis, MN, USA (e-mail: ingra107@umn.edu).

Author Contribution Concept and design: All authors Acquisition, analysis, or interpretation of data: Ingraham, Usher, Tignanelli
Drafting of the manuscript: All authors

Critical revision of the manuscript for important intellectual content: All authors

Reprints will not be available from the authors. All authors significantly contributed to developing, writing, and revising this manuscript.

Funding

1. NIH NHLBI T32HLO7741 (NEI)

2. AHRQ RO1HS24532 (GBM)

3. AHRQ R01HS26732 (MGU)

4. AHRQ/PCORI K12HSO26379 (CJT)

4. NIH NCATS KL2TROO2492, UL1TR002494

\section{Declarations:}

Conflict of Interest: The authors declare that they do not have a conflict of interest.

\section{REFERENCES}

1. Balsa AI, Seiler N, McGuire TG, Bloche MG. Clinical uncertainty and healthcare disparities. Am JL \& Med 2003;29:203.

2. HealthPeople.gov 2020;Pages. Accessed at Office of Disease Prevention and Health Promotion at https://www.healthypeople.gov/2020/topicsobjectives/topic/social-determinants-of-health on 8/25/2020 2020.

3. Wang $\mathbf{Z}$, Tang $\mathbf{K}$. Combating COVID-19: health equity matters. Nat Med 2020;26(4):458.

4. Laster Pirtle WN. Racial capitalism: a fundamental cause of novel coronavirus (COVID-19) pandemic inequities in the United States. Health Educ Behav 2020;47(4):504-8.

5. Meneses-Navarro S, Freyermuth-Enciso MG, Pelcastre-Villafuerte BE, Campos-Navarro R, Melendez-Navarro DM, Gomez-Flores-Ramos L. The challenges facing indigenous communities in Latin America as they confront the COVID-19 pandemic. Int $\mathrm{J}$ Equity Health 2020; 19(1):63.

6. Wadhera RK, Wadhera P, Gaba P, Figueroa JF, Maddox KEJ, Yeh RW, et al. Variation in COVID-19 hospitalizations and deaths across New York City boroughs. JAMA. 2020;323(21):2192-5.

7. Adhikari S, Pantaleo NP, Feldman JM, Ogedegbe O, Thorpe L, Troxel AB. Assessment of community-level disparities in coronavirus disease 2019 (COVID-19) infections and deaths in large US metropolitan areas. JAMA Netw Open. 2020;3(7):e2016938-e.

8. Duran DG, Pérez-Stable EJ. Novel Approaches to Advance Minority Health and Health Disparities Research. Am J Public Health 2019;109(S1):S8-S10.

9. Lopez L, 3rd, Hart LH, 3rd, Katz MH. Racial and ethnic health disparities related to COVID-19. JAMA. 2021;325(8):719-20.

10. Polyakova M, Udalova V, Kocks G, Genadek K, Finlay K, Finkelstein AN. Racial disparities in excess all-cause mortality during the early COVID-19 pandemic varied substantially across states: study examines the geographic variation in excess all-cause mortality by race to better understand the impact of the COVID-19 pandemic. Health Aff 2021;40(2):307-16.

11. Baltrus PT, Douglas M, Li C, Caplan LS, Blount M, Mack D, et al. Percentage of Black population and primary care shortage areas associated with higher COVID-19 case and death rates in Georgia Counties. South Med J 2021;114(2):57-62.

12. Tan SB, deSouza P, Raifman M. Structural racism and COVID-19 in the USA: a county-level empirical analysis. J Racial Ethn Health Disparities. 2021:1-11. https://doi.org/10.1007/s40615-020-00948-8.

13. Starr LT, O'Connor NR, Meghani SH. Improved serious illness communication may help mitigate racial disparities in care among Black Americans with COVID-19. J Gen Intern Med. 2021;36(4):1071-1076.

14. Dalsania AK, Fastiggi MJ, Kahlam A, Shah R, Patel K, Shiau S, et al. The relationship between social determinants of health and racial disparities in COVID-19 mortality. J Racial Ethn Health Disparities. 2021;5:1-8. https://doi.org/10.1007/s40615-020-00952-y

15. Carethers JM. Insights into disparities observed with COVID-19. Journal of internal medicine. 2021;289(4):463-73. 
16. Bui CN, Peng C, Mutchler JE, Burr JA. Race and ethnic group disparities in emotional distress among older adults during the COVID19 pandemic. The Gerontologist. 2021;61(2):262-72.

17. Tiako MJN, Forman HP, Nunez-Smith M. Racial health disparities, COVID-19, and a way forward for US health systems. J Hosp Med 2021;16(1):50-2.

18. Raisi-Estabragh Z, McCracken C, Bethell MS, Cooper J, Cooper C, Caulfield MJ, et al. Greater risk of severe COVID-19 in Black, Asian and Minority Ethnic populations is not explained by cardiometabolic, socioeconomic or behavioural factors, or by $25(\mathrm{OH})$-vitamin D status: study of 1326 cases from the UK Biobank. Journal of Public Health. 2020;42(3):451-60

19. Rogers TN, Rogers CR, VanSant-Webb E, Gu LY, Yan B, Geadan F. Racial disparities in COVID-19 mortality among essential workers in the United States. World medical \& health policy 2020:https://doi.org/10. 1002/wmh3.358

20. Joseph NP, Reid NJ, Som A, Li MD, Hyle EP, Dugdale CM, et al. Racial/ ethnic disparities in disease severity on admission chest radiographs among patients admitted with confirmed COVID-19: a retrospective cohort study. Radiology. 2020;297(3):E303-E12

21. Sentell T, Braun KL. Low health literacy, limited English proficiency, and health status in Asians, Latinos, and other racial/ethnic groups in California. J Health Commun 2012; 17(sup3):82-99.

22. Rozenfeld Y, Beam J, Maier H, Haggerson W, Boudreau $\mathbf{K}$, Carlson $\mathbf{J}$, et al. A model of disparities: risk factors associated with COVID-19 infection. Int J Equity Health 2020;19(1):126.

23. Mackey $\mathbf{K}$, Ayers CK, Kondo KK, Saha S, Advani SM, Young S, et al. Racial and ethnic disparities in COVID-19-related infections, hospitalizations, and deaths: a systematic review. Annals of internal medicine. 2021;174(3):362-73.

24. Escobar GJ, Adams AS, Liu VX, Soltesz L, Chen YI, Parodi SM, et al. Racial disparities in COVID-19 testing and outcomes: retrospective cohort study in an integrated health system. Ann Intern Med. 2021. https://doi.org/10.7326/M20-6979.

25. Anyane-Yeboa A, Sato T, Sakuraba A. Racial disparities in COVID-19 deaths reveal harsh truths about structural inequality in America. J Intern Med 2020;288(4):479-80.

26. Azar KM, Shen Z, Romanelli RJ, Lockhart SH, Smits K, Robinson S, et al. Disparities in outcomes among COVID-19 patients in a large health care system in California: study estimates the COVID-19 infection fatality rate at the US county level. Health Aff 2020;39(7):1253-62.

27. Blitz MJ, Rochelson B, Prasannan L, Shan W, Chervenak FA, Nimaroff M, et al. Racial and ethnic disparity and spatiotemporal trends in severe acute respiratory syndrome coronavirus 2 prevalence on obstetrical units in New York. Am J Obstet Gynecol MFM. 2020;2(4):100212

28. Caraballo C, McCullough M, Fuery MA, Chouairi F, Keating C Ravindra NG, et al. COVID-19 infections and outcomes in a live registry of heart failure patients across an integrated health care system. PLoS One 2020;15(9):e0238829.

29. Gold JA. Characteristics and clinical outcomes of adult patients hospitalized with COVID-19-Georgia, March 2020. MMWR. Morbidity and mortality weekly report. 2020;69.

30. Azar KMJ, Shen Z, Romanelli RJ, Lockhart SH, Smits K, Robinson S, et al. Disparities in outcomes among COVID-19 patients in a large health care system in California. Health Aff (Millwood) 2020;39(7):1253-62.

31. Munoz-Price LS, Nattinger AB, Rivera F, Hanson R, Gmehlin CG, Perez A, et al. Racial disparities in incidence and outcomes among patients with COVID-19. JAMA Netw Open 2020;3(9):e2021892.

32. Price-Haywood EG, Burton J, Fort D, Seoane L. Hospitalization and mortality among Black patients and White patients with COVID-19. N Engl J Med 2020;382(26):2534-43.

33. Karmakar M, Lantz PM, Tipirneni R. Association of social and demographic factors with COVID-19 incidence and death rates in the US. JAMA Netw Open 202 1;4(1):e2036462.

34. Hooper MW, Nápoles AM, Pérez-Stable EJ. COVID-19 and racial/ethnic disparities. Jama. 2020

35. Singh GK. Area deprivation and widening inequalities in US mortality, 1969-1998. Am J Public Health 2003;93(7):1137-43.

36. Knighton AJ, Savitz L, Belnap T, Stephenson B, VanDerslice $\mathbf{J}$ Introduction of an area deprivation index measuring patient socioeconomic status in an integrated health system: implications for population health. EGEMS. 2016;4(3): 1238.

37. Patel AP, Paranjpe MD, Kathiresan NP, Rivas MA, Khera AV. Race, socioeconomic deprivation, and hospitalization for COVID-19 in English participants of a national biobank. Int J Equity Health 2020;19(1):114.
38. Niedzwiedz CL, O'Donnell CA, Jani BD, Demou E, Ho FK, CelisMorales C, et al. Ethnic and socioeconomic differences in SARS-CoV-2 infection: prospective cohort study using UK Biobank. BMC Med 2020;18(1): 160 .

39. Das A, Ghosh S, Das K, Basu T, Das M, Dutta I. Modeling the effect of area deprivation on COVID-19 incidences: a study of Chennai megacity, India Public Health 2020;185:266-9.

40. Elixhauser A, Steiner C, Harris DR, Coffey RM. Comorbidity measures for use with administrative data. Med Care 1998:8-27.

41. Office of Community and Rural Affairs 2019;Pageshttps:// pcrd.purdue.edu/ruralindianastats/geographic-classifications.php on 8/25/2020 2020.

42. Jakobsen JC, Gluud C, Wetterslev J, Winkel P. When and how should multiple imputation be used for handling missing data in randomised clinical trials - a practical guide with flowcharts. BMC Med Res Methodol 2017; 17(1): 162 .

43. Zhou F, Yu T, Du R, Fan G, Liu Y, Liu Z, et al. Clinical course and risk factors for mortality of adult inpatients with COVID-19 in Wuhan, China: a retrospective cohort study. Lancet. 2020;395(10229): 1054-62.

44. Ingraham NE, Lotfi-Emran S, Thielen BK, Techar K, Morris RS, Holtan SG, et al. Immunomodulation in COVID-19. Lancet Respir Med 2020;8(6):544-6.

45. Bailey ZD, Krieger N, Agenor M, Graves J, Linos N, Bassett MT Structural racism and health inequities in the USA: evidence and interventions. Lancet. 2017;389(10077): 1453-63.

46. Figueroa JF, Wadhera RK, Mehtsun WT, Riley K, Phelan J, Jha AK Association of race, ethnicity, and community-level factors with COVID19 cases and deaths across US counties. Healthcare, 2021;9(1):100495. https://doi.org/10.1016/j.hjdsi.2020.100495.

47. Du XL, Fang S, Vernon SW, El-Serag H, Shih YT, Davila J, et al. Racial disparities and socioeconomic status in association with survival in a large population-based cohort of elderly patients with colon cancer. Cancer. 2007;110(3):660-9.

48. Parise CA, Caggiano V. Disparities in race/ethnicity and socioeconomic status: risk of mortality of breast cancer patients in the California Cancer Registry, 2000-2010. BMC Cancer 2013;13(1):449.

49. Du XI, Fang SY, Meyer TE. Impact of treatment and socioeconomic status on racial disparities in survival among older women with breast cancer. American Journal of Clinical Oncology-Cancer Clinical Trials 2008;31(2):125-32.

50. Fuller-Thomson E, Nuru-Jeter A, Minkler M, Guralnik JM Black-White disparities in disability among older Americans: further untangling the role of race and socioeconomic status. Journal of aging and health 2009;21(5):677-98.

51. Yehia BR, Winegar A, Fogel R, Fakih M, Ottenbacher A, Jesser C, et al Association of race with mortality among patients hospitalized with coronavirus disease 2019 (COVID-19) at 92 US hospitals. JAMA Netw Open 2020;3(8):e2018039.

52. Khanijahani A, Tomassoni L. Socioeconomic and racial segregation and COVID-19: concentrated disadvantage and black concentration in association with COVID-19 deaths in the USA. J Racial Ethn Health Disparities 2021:1-9.

53. Eisner MD, Blanc PD, Omachi TA, Yelin EH, Sidney S, Katz PP, et al. Socioeconomic status, race and COPD health outcomes. J Epidemiol Community Health 2011;65(1):26-34.

54. Ejike CO, Woo H, Galiatsatos P, Paulin LM, Krishnan JA, Cooper CB, et al. Contribution of individual and neighborhood factors to racial disparities in respiratory outcomes. Am J Respir Crit Care Med. 2021;203(8):987-97.

55. Williamson EJ, Walker AJ, Bhaskaran $\mathbf{K}$, Bacon S, Bates C, Morton CE, et al. Factors associated with COVID-19-related death using OpenSAFELY. Nature. 2020;584(7821):430-6.

56. Ogedegbe G, Ravenell J, Adhikari S, Butler M, Cook T, Francois F, et al. Assessment of racial/ethnic disparities in hospitalization and mortality in patients with COVID-19 in New York city. JAMA Netw Open 2020;3(12):e2026881-e.

57. Dorn AV, Cooney RE, Sabin ML. COVID-19 exacerbating inequalities in the US. Lancet. 2020;395(10232):1243-4.

58. Zhang W, Hong S, Takeuchi DT, Mossakowski KN. Limited English proficiency and psychological distress among Latinos and Asian Americans. Soc Sci Med 2012;75(6):1006-14.

59. Ingraham NE, Boulware D, Sparks MA, Schacker T, Benson B, Sparks JA, et al. Shining a light on the evidence for hydroxychloroquine in SARSCoV-2. Crit Care 2020;24(1):182 
60. Ingraham NE, Tignanelli CJ. Fact versus science fiction: fighting coronavirus disease 2019 requires the wisdom to know the difference. Crit Care Explor 2020;2(4):e0108.

61. Patzer RE, McClellan WM. Influence of race, ethnicity and socioeconomic status on kidney disease. Nat Rev Nephrol 2012;8(9):533.

62. Koch S, Yoon L, Gils B. From the exposome to the socioexposome in COVID-19 research-a call for more multidisciplinary research. JAMA Netw Open 2020;3(12):e2032287-e.

63. Peek ME, Simons RA, Parker WF, Ansell DA, Rogers SO, Edmonds BT. COVID-19 among African Americans: an action plan for mitigating disparities. American Journal of Public Health 2021; 111: 286-292.

64. Stephenson J. Despite COVID-19's toll on people of color, many don't view racism as a factor in health outcomes. JAMA Health Forum, 2021. American Medical Association: e210036-e.

65. Bruce L, Tallman $\mathbf{R}$. Promoting racial equity in COVID-19 resource allocation. J Med Ethics 2021.

66. Ahmed F, Ahmed N, Pissarides C, Stiglitz J. Why inequality could spread COVID-19. Lancet Public Health 2020;5(5):e240.

67. Alsan M, Stantcheva S, Yang D, Cutler D. Disparities in coronavirus 2019 reported incidence, knowledge, and behavior among US adults. JAMA Netw Open 2020;3(6):e2012403-e.

68. Bell CN, Sacks TK, Tobin CST, Thorpe Jr RJ. Racial non-equivalence of socioeconomic status and self-rated health among African Americans and Whites. SSM-population health 2020;10:100561.
69. Prasad SJ, Nair P, Gadhvi K, Barai I, Danish HS, Philip AB. Cultural humility: treating the patient, not the illness. Med Educ Online. 2016 ;21:30908. https://doi.org/10.3402/meo.v21.30908.

70. White DB, Angus DC. A proposed lottery system to allocate scarce COVID-19 medications: promoting fairness and generating knowledge. Jama. 2020;324(4):329-30.

71. Seaman J 2021;Pages. Accessed at Denver Post at https://www. denverpost.com/2021/01/22/colorado-covid-19-vaccine-racial-inequity/ on $1 / 22 / 20212021$.

72. 2021;Pages. Accessed at Government of the District of Columbia at https://coronavirus.dc.gov/data on 1/22/2021 2021.

73. Wallis C. Why racism, not race, is a risk factor for dying of COVID-19. Scientific American. 2020. Available at: https://www.scientificamerican. com/article/why-racism-not-race-is-a-risk-factor-for-dying-of-covid$191 /$.

Publisher's Note Springer Nature remains neutral with regard to jurisdictional claims in published maps and institutional affiliations. 